\title{
Urinary exosomal vitronectin predicts vesicoureteral reflux in patients with neurogenic bladders and spinal cord injuries
}

\author{
JUE LI $^{1,2}$, SHIYING CAI ${ }^{1}$, CHUNXIAN ZENG $^{1}$, LING CHEN $^{1}$, CHUN ZHAO $^{1}$, YING HUANG $^{1}$ and WENZHI CAI ${ }^{1,2}$ \\ ${ }^{1}$ Department of Nursing, Shenzhen Hospital, Southern Medical University, Shenzhen, Guangdong 518101; \\ ${ }^{2}$ School of Nursing, Southern Medical University, Guangzhou, Guangdong 510515, P.R. China
}

Received August 29, 2021; Accepted October 29, 2021

DOI: $10.3892 / \mathrm{etm} .2021 .10988$

\begin{abstract}
Neurogenic bladder (NGB) is an important complication of urinary tract dysfunction after spinal cord injury (SCI). However, using urodynamics and urography to guide therapy remains invasive and complicated. Therefore, the present study aimed to identify potential noninvasive biomarkers from urinary exosomes that can facilitate diagnosis and guide prognosis of patients with NGB subsequent to SCI. Urinary exosomes were isolated, and their proteome profile was analyzed by mass spectrometry. Transmission electron microscopy and Nanoparticle Tracking Analysis confirmed the size and morphological characteristics of urinary exosomes. In addition, bioinformatics analysis and parallel reaction monitoring (PRM) were used to screen candidate biomarkers. The selected biomarkers were validated using western blotting and ELISA. Mass spectrometry identified 134 upregulated proteins and 99 downregulated proteins between the vesicoureteral reflux (VUR) and non-VUR groups. A total of 18 candidate proteins were selected for PRM validation, but only vitronectin (VTN) and $\alpha-1$ type I collagen (COL1A1) demonstrated significant differences. In the validation experiments using western blotting and ELISA, VTN was exclusively highly expressed in VUR patients compared with non-VUR patients. However, the ELISA results of COL1A1 revealed no significant difference when a larger sample size was used. Furthermore, a receiver operating characteristic curve of ELISA-based VTN demonstrated an area under the curve of 0.795 and $80 \%$ sensitivity at a threshold set to give
\end{abstract}

Correspondence to: Professor Wenzhi Cai, Department of Nursing, Shenzhen Hospital, Southern Medical University, 1333 Xinhu Road, Baoan, Shenzhen, Guangdong 518101, P.R. China

E-mail: caiwzh@smu.edu.cn

Abbreviations: NGB, neurogenic bladder; SCI, spinal cord injury; TEM, transmission electron microscopy; NTA, Nanoparticle Tracking Analysis; PRM, parallel reaction monitoring; VUR, vesicoureteral reflux

Key words: lower urinary tract damage, neurogenic bladder, spinal cord injury, vesicoureteral reflux, vitronectin
$82.9 \%$ specificity. Collectively, these results suggested that VTN in urinary exosomes may be used as a biomarker to predict the progression and guide the prognosis of NGB.

\section{Introduction}

Spinal cord injuries (SCI) are defined as acute or chronic damage to the spinal cord, respectively traumatic or caused by comorbidities (1). SCI are irreversible (2) and patients suffer from several complications (3), including urinary tract infection and neurogenic bladder (NGB) (4). It is reported that $46 \%$ of NGB were caused by SCI (5). NGB refers to the dysfunction of bladder secondary to any neurological disease, including SCI (6). NGB results in elevated detrusor pressure (7) and continuous vesicoureteral reflux (VUR), which causes a series of damage to the upper urinary tract, including ureter dilation, hydronephrosis, kidney failure and even patients' death (8). Therefore, it is important to identify VUR early and perform an active and effective intervention to reduce or delay the occurrence of VUR and prevent upper urinary tract damage, which may improve the prognosis of patients with NGB and have a positive effect on improving patients' quality of life.

Exosomes are small, cell-secreted vesicles ranging in size from 30-100 nm (9). Exosomes contain a variety of biomolecules, including proteins, mRNAs, long non-coding RNAs and microRNAs (10), which not only reflect the functional state of exosome-derived cells but also affect the biological function of downstream target cells (11). As promising diagnostic candidates, exosomes have been evaluated in several types of cancer, including bladder (12) and renal cancer (13). Urinary exosomes can originate from the kidney, ureter, bladder or even prostate. Therefore, urine exosomal proteins may contain important biological information of disease pathophysiology (14) and can be used as non-invasive and convenient potential markers (15). Some exosome-related studies have been performed on non-cancerous urinary diseases, such as renal injury (16), $\operatorname{Ig} \mathrm{A}$ nephropathy (17) and urinary tract infection (18). However, to the best of our knowledge, no research has investigated urinary exosome proteins in patients with NGB after SCI.

Accordingly, the present study was designed to identify the different protein profiles of urine exosomes between VUR and non-VUR patients, plus to explore potential biomarkers in predicting diagnosis and guiding prognosis in patients with SCI. 


\section{Materials and methods}

Patients and experimental design. Multicenter patients with SCI were recruited into the present study from September 2019 to November 2020. A total of 316 patients (age, 5-64, male, 182; female, 134) were initiatively recruited. After screening, only 60 patients (male, 45; female, 15) were included in the present study. Patient inclusion screening criteria were as follows: i) Patients met the ASIA diagnostic criteria (19) and subsequently had NGB symptoms; ii) patients were aged over 18 years old regardless of sex; iii) patients had no significant urinary tract infections or symptoms of hematuria; and iv) patients had no serious complications of other organs. The exclusion criteria included the following: i) Patients with diabetes or hypertension disease; ii) patients with severe chronic heart and lung disease or with chronic liver, kidney and urinary system disease before being investigated; and iii) patients with severe infectious diseases. According to the urological ultrasound or urography results, the included 60 patients were divided into either the VUR group or non-VUR group. The guideline flowchart of the research protocol is presented in Fig. 1. To illustrate the differential exosome protein profile and to select promising predictable biomarkers between VUR and non-VUR patients, a group of 15 VUR patients and 15 non-VUR patients were enrolled in the preliminary screening study. Subsequently, five samples were collected from each group for preliminary mass spectrometry analysis, and then another 10 samples were used for target analysis by parallel reaction monitoring (PRM). In the validation study, a total of 25 patients with VUR and 35 non-VUR patients were enrolled (including the patients in the first screening part).

The present study was conducted by Shenzhen Hospital of Southern Medical University where the study center is located. The participating hospitals included Shenzhen Hospital of Southern Medical University (Shenzhen, China), Shenzhen Xiao Chuanguo Hospital (Shenzhen, China), Shenzhen Longcheng Hospital (Shenzhen, China), Nanfang Hospital of Southern Medical University (Guangzhou, China), People's Hospital of Mianzhu, Sichuan Province (Mianzhu, China) and Bayi Rehabilitation Centre of Sichuan Province (Chengdu, China). The study protocol was approved by the Scientific Ethics Committee of Shenzhen Hospital of Southern Medical University (approval no. NYSZYYEC20180002). All participants provided written informed consent according to the principles of the Helsinki Declaration. All data were kept confidential and processed anonymously.

Exosome isolation and identification. Urinary exosomes were isolated by serial centrifugation as previously described (18). Briefly, urine samples were packed with sterile centrifuge tubes and then transported to the laboratory by icebox. Urine samples were passed through a $0.22-\mu \mathrm{m}$ polyvinylidene difluoride filter and subjected to ultracentrifugation at $170,000 \times \mathrm{x} \mathrm{g}, 4^{\circ} \mathrm{C}$, for $60 \mathrm{~min}$. After washing in PBS, exosomes were resuspended in cell lysis buffer (containing $150 \mathrm{mM} \mathrm{NaCl}, 20 \mathrm{mM}$ Tris-HCl pH 7.5 and 1\% Triton X-100; cat. no. P0013; Beyotime Institute of Biotechnology) for immediate further use or stored at $-80^{\circ} \mathrm{C}$. Before being used, the amount of protein was measured using the Pierce ${ }^{\mathrm{TM}}$ Rapid Gold BCA assay kit (cat. no. A53225;
Thermo Fisher Scientific, Inc.) according to the manufacturer's instructions.

Transmission electron microscopy (TEM) and Nanoparticle Tracking Analysis (NTA) were performed. After being fixed in $4 \%$ formaldehyde for $10 \mathrm{~min}$ and deposited onto a copper grid, the exosome samples were transferred into $1 \%$ glutaraldehyde in PBS for 5 min and stained with $2.0 \%$ uranyl acetate in aqueous suspension for $2 \mathrm{~min}$. All the aforementioned procedures were performed at room temperature unless clearly stated. Finally, the JEM1400 (JEOL, Ltd.) electron microscope was used at $80 \mathrm{kV}$ to observing and capture images. For NTA, NanoSight NS3000 with $405 \mathrm{~nm}$ blue laser (Malvern Panalytical, Ltd.) was used. Measurement data were analyzed using NTA 3.0 analysis software (Malvern Panalytical, Ltd.).

Tandem mass tag spectrometry analysis and PRM. Tandem mass tag (TMT) labeling quantitative proteomics analyses were carried out on an EASY-nLC 1000 UPLC system (Thermo Fisher Scientific, Inc.). A total of $100 \mu \mathrm{g}$ of protein for each sample was digested with trypsin at 1:50 trypsin-to-protein mass ratio for the first digestion overnight $\left(37^{\circ} \mathrm{C}\right)$ and 1:100 trypsin-to-protein mass ratio for a second 4 h-digestion $\left(37^{\circ} \mathrm{C}\right)$. After trypsin digestion, desalted peptide was reconstituted in $0.5 \mathrm{M}$ TEAB and processed according to the manufacturer's protocol for the TMT10plex ${ }^{\mathrm{TM}}$ Isobaric Label Reagent Set kit (cat. no. 90406; Thermo Fisher Scientific, Inc.). The tryptic peptides were fractionated into fractions by high-pH reverse-phase high-performance liquid chromatography using BETASIL ${ }^{\mathrm{TM}}$ PREP C18 HPLC Columns (cat. no. 70105-259070A; Thermo Fisher Scientific, Inc.). The gradient consisted of an increase from 6 to $23 \%$ solvent B ( $0.1 \%$ formic acid in $98 \%$ acetonitrile) over $26 \mathrm{~min}, 23$ to $35 \%$ in $8 \mathrm{~min}$ and 35 to $80 \%$ in $3 \mathrm{~min}$, then holding at $80 \%$ for the last $3 \mathrm{~min}$, all at a constant flow rate of $400 \mathrm{nl} / \mathrm{min}$. The electrospray voltage applied was $2.0 \mathrm{kV}$. The $\mathrm{m} / \mathrm{z}$ scan range was 350 to 1800 for full scan and intact peptides were detected in the Orbitrap at a resolution of 70,000. A data-dependent procedure that alternated between one MS scan followed by $20 \mathrm{MS} / \mathrm{MS}$ scans with 15.0 s dynamic exclusion. Automatic gain control was set at 5E4. Fixed first mass was set as $100 \mathrm{~m} / \mathrm{z}$.

The resulting peptides were subjected to a nanoelectrospray ionization source (Nanospray Flex ${ }^{\mathrm{TM}}$ Ion Source; cat. no. ES071; Thermo Fisher Scientific, Inc.) followed by tandem mass spectrometry (MS/MS) in Q Exactive ${ }^{\mathrm{TM}}$ Plus (Thermo Fisher Scientific, Inc.) coupled online to the UPLC. Peptides were then selected for MS/MS using NCE setting at 28 and the fragments were detected in the Orbitrap at a resolution of 17,500. The resulting MS/MS data were processed using Maxquant search engine v.1.5.2.8 (https://maxquant.net/). The mass tolerance for precursor ions was set as $20 \mathrm{ppm}$ in the First search and $5 \mathrm{ppm}$ in the Main search, and the mass tolerance for fragment ions was set as $0.02 \mathrm{Da}$. The false discovery rate of peptide identifications was adjusted to $<1 \%$ and the minimum score for modified peptides was set to $>40$.

According to the TMT results, candidate proteins containing $\geq 2$ unique peptides were designed for PRM. The unique peptides were used to identify the target proteins. Enzymatic digestion is an important step to obtain peptides. However, some proteins were quantified as containing only one peptide due to the experimental error in enzyme digestion, which resulted in 


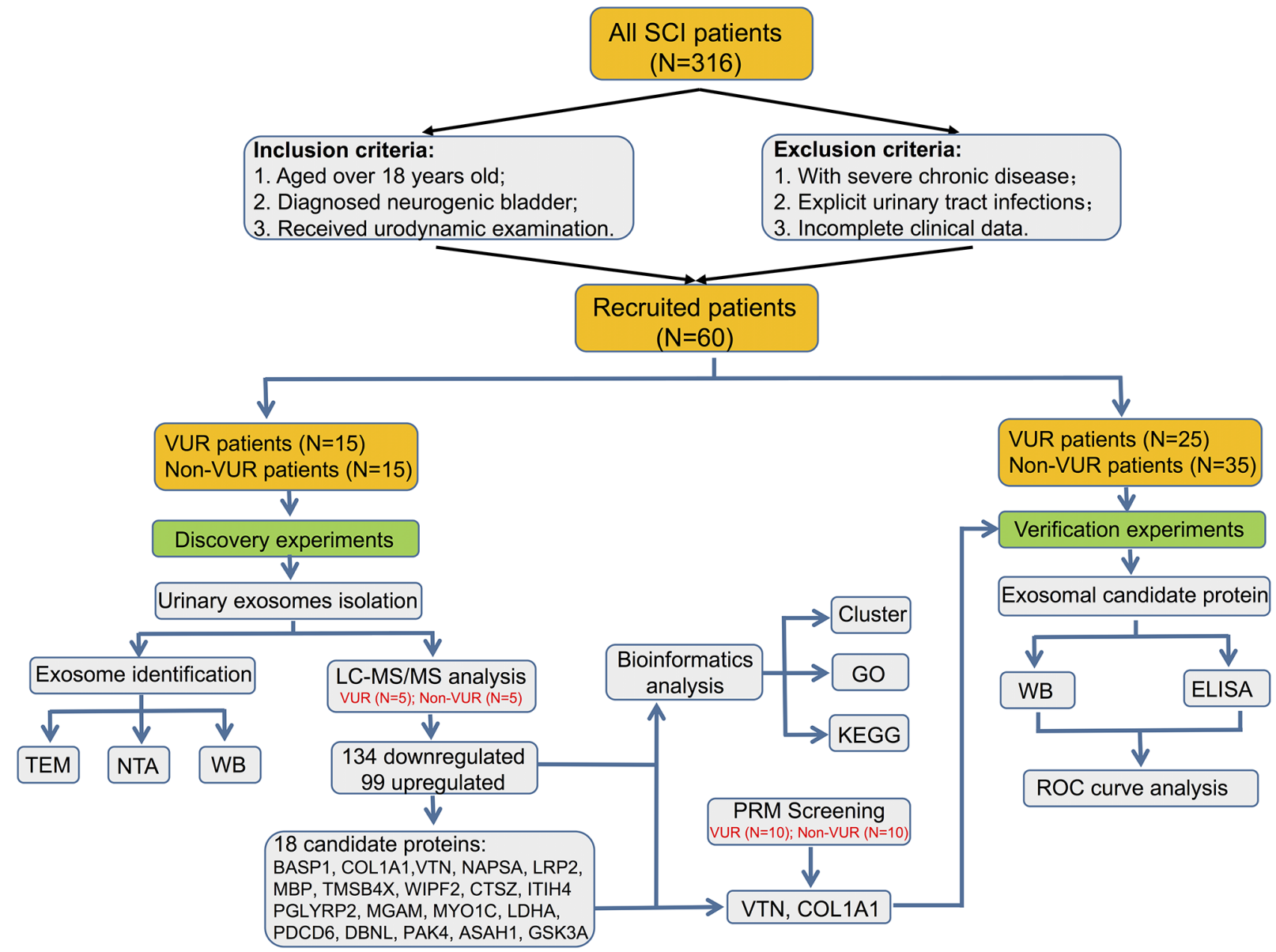

Figure 1. Workflow of the present study design and brief experimental schedule. SCI, Spinal cord injury; VUR, vesicoureteral reflux; non-VUR, non-vesicoureteral reflux; LC-MS/MS, liquid chromatography-mass spectrometry; PRM, Parallel Reaction Monitoring; TEM, Transmission Electron Microscope; NTA, Nanoparticle Tracking Analysis; WB, Western blotting; GO, Gene Ontology; KEGG, Kyoto Encyclopedia of Genes and Genomes; ROC, receiver operating characteristic.

a lower abundance of unique peptides. A total of 10 samples were included in each group. The protein of each sample was enzymatically hydrolyzed with an equal amount of standard protein, the volume was adjusted to the same with lytic solution and then dithiothreitol was added to reduce the final concentration to $5 \mathrm{mM}$ at $56^{\circ} \mathrm{C}$ for $30 \mathrm{~min}$. The following procedure on Q Exactive ${ }^{\mathrm{TM}}$ Plus was the same as the TMT as aforementioned. Fragment ion peak areas of the selected peptides were used for quantitative analysis. The quantitative data processing and proteomic analysis were processed using Skyline v.3.6 (http://proteome.gs.washington.edu/software/skyline).

Bioinformatics analysis. In the bioinformatics analysis of TMT results, Gene Ontology (GO) annotation proteome was derived from the UniProt-GOA database (http://www.ebi. ac.uk/GOA/). Then identified proteins were classified into three categories: biological process, cellular component and molecular function. Furthermore, Clusters of Orthologous Groups/euKaryotic Ortholog Groups of proteins (COG/KOG) categories database (version '2003 COGs, 2014 update'; https://www.ncbi.nlm.nih.gov/research/cog-project/) was used to identify the functional annotation of differential proteins. Pathways were annotated using the Kyoto Encyclopedia of Genes and Genomes database (Version, 'Release 92.0, October 1, 2019'; http://www.genome.jp/kegg/). In the bioinformatics analysis of PRM results, Gene function enrichment (FunRich) analysis and Gene Ontology analysis of candidate proteins were performed using the GenCLiP platform 3.0 (20).

Western blotting. Typical loading controls such as cytoskeletal elements (tubulin/actin) or metabolic enzymes (GAPDH) are lacking in exosome samples (21). Quantification of total protein is more effective and reliable compared with typical housekeeping genes as loading controls (22). Therefore, the present study used total protein as a loading control by Coomassie G250 (cat. no. ST030; Beyotime Institute of Biotechnology). Exosome samples were diluted in cell lysis buffer (cat. no. P0013; Beyotime Institute of Biotechnology) to extract proteins. Protein concentrations were measured using the BCA method. A total of $5 \mu \mathrm{g}$ protein was loaded in each lane, separated using $12 \%$ SDS-PAGE and electrically transferred to PVDF membranes. After blocking for $1 \mathrm{~h}$ at room temperature with $5 \%$ free-fat milk diluted with $0.2 \%$ Tween- 20 in PBS, the membranes were incubated at $4^{\circ} \mathrm{C}$ overnight with rabbit anti-vitronectin antibody (1:5,000; cat. no. ab45139; Abcam), rabbit anti- $\alpha-1$ type I collagen (COL1A1) antibody (1:1,000; cat. no. 72026; Cell Signaling Technology, Inc.), rabbit anti-Alix antibody (1:1,000; cat. no. ab88388; Abcam) or rabbit anti-CD63 antibody (1:5,000; cat. no. ab134045; Abcam). Goat anti-rabbit horseradish peroxidase (HRP)-conjugated 
Table I. Clinical characteristics between the VUR and non-VUR groups.

\begin{tabular}{|c|c|c|c|c|}
\hline Parameter & VUR (n=25) & Non-VUR $(n=35)$ & $\mathrm{X}^{2}$ or t-test & P-value \\
\hline $\operatorname{Sex}(n, \%)$ & & & 1.120 & $0.290^{\mathrm{a}}$ \\
\hline Male & 17 & 28 & & \\
\hline Female & 8 & 7 & & \\
\hline Age, years & $34.64 \pm 12.98$ & $40.63 \pm 12.42$ & -1.807 & 0.076 \\
\hline Course of diseases, days & $540.44 \pm 533.04$ & $167.09 \pm 297.28$ & 3.464 & 0.001 \\
\hline Damage level & & & & $0.156^{\mathrm{b}}$ \\
\hline Cervical spine & 6 & 12 & & \\
\hline Thoracic vertebrae & 8 & 15 & & \\
\hline Lumbar spine & 8 & 3 & & \\
\hline Cauda equina & 3 & 5 & & \\
\hline AISA level & & & & $0.128^{\mathrm{b}}$ \\
\hline A & 9 & 20 & & \\
\hline B & 4 & 3 & & \\
\hline $\mathrm{C}$ & 10 & 6 & & \\
\hline $\mathrm{D}$ & 2 & 6 & & \\
\hline Urination methods & & & 1.714 & $0.190^{\mathrm{a}}$ \\
\hline Automatic/Leakage & 7 & 5 & & \\
\hline Catheterization/Cystostomy & 18 & 30 & & \\
\hline Bladder compliance & & & & $1.44 \times 10^{-4 b}$ \\
\hline Normal & 0 & 14 & & \\
\hline Abnormal & 25 & 21 & & \\
\hline Residual bladder urine, $\mathrm{ml}$ & $139.20 \pm 82.86$ & $78.54 \pm 41.93$ & 3.723 & $4.48 \times 10^{-4}$ \\
\hline Safe bladder capacity, ml & $295.20 \pm 75.17$ & $342.29 \pm 167.12$ & -1.315 & 0.879 \\
\hline Intravesical pressure, $\mathrm{cm} \mathrm{H}_{2} \mathrm{O}$ & $95.56 \pm 23.53$ & $40.46 \pm 21.60$ & 9.386 & $3.08 \times 10^{-13}$ \\
\hline
\end{tabular}

${ }^{a}$ Chi-square and ${ }^{b}$ Fisher's exact tests were used. Counting values are expressed as count (percentage). Measurement data are expressed as means \pm SD. VUR, vesicoureteral reflux; AISA, American Spinal Injury Association.

secondary antibodies (1:5,000; cat. no. 7074S; Cell Signaling Technology, Inc.) were co-incubated for $1 \mathrm{~h}$ at room temperature. Signals were detected and captured in the Bio-Rad ChemiDoc Touch Imaging System (Bio-Rad Laboratories, Inc.) using Pierce ${ }^{\mathrm{TM}}$ ECL Western Blotting Substrate (Thermo Fisher Scientific, Inc.). Optical density values were determined using ImageJ v1.53a software (National Institutes of Health).

ELISA. A modified lysis method was performed as described previously (23). Notably, the amount of protein in exosomes resuspended in PBS was measured using a BCA assay kit (cat. no. ZJ101; Epizyme, Inc.). Subsequently, similar protein amounts of urinary exosomes were dissolved in a lysis buffer (cat. no. P0013; Beyotime Institute of Biotechnology). To detect exosomal vitronectin (VTN) and COL1A1, $10 \mu \mathrm{g}$ total protein was used. Human VTN ELISA (cat. no. CSB-E08983h; CUSABIO Technology LLC) and human COL1A1 ELISA (cat. no. RK01149; ABclonal Biotech Co., Ltd.) kits were used following the manufacturer's protocol.

Statistical analysis. Statistical analysis was conducted using SPSS version 18.0 (SPSS, Inc.) and GraphPad Prism 9 (GraphPad Software, Inc.). Bioinformatics figures in the LC-MS/MS results were exported by R studio (v1.3.1093;
RStudio, Inc.) (24). The homogeneity tests between the VUR and non-VUR groups were validated using chi-square, Fisher's exact or t-tests. Comparison between two groups was made using paired t-test or Mann-Whitney U test. Receiver operating characteristic (ROC) curves were used to calculate the overall diagnostic performance of candidate biomarkers. The data are presented as means $\pm \mathrm{SD}$. All statistical tests were two-tailed, and $\mathrm{P}<0.05$ was considered to indicate a statistically significant difference. All the validation experiments were performed in triplicates.

\section{Results}

Socio-demographic and clinical characteristics. Clinical data from the VUR and non-VUR groups were summarized in Table I. In total, 25 VUR patients and 35 non-VUR patients were included in the present study. No significant difference appeared in age, sex ratio, injury region, AIS level and urination methods between the two groups (Table I), suggesting statistical comparability between the two groups. As expected, the course of diseases, residual bladder urine and intravesical pressure in the VUR group were significantly higher compared with the non-VUR group (Table I). Notably, the bladder safe-capacity $(295.20 \pm 75.17 \mathrm{ml}$ vs. $342.29 \pm 167.12 \mathrm{ml})$ revealed 
A

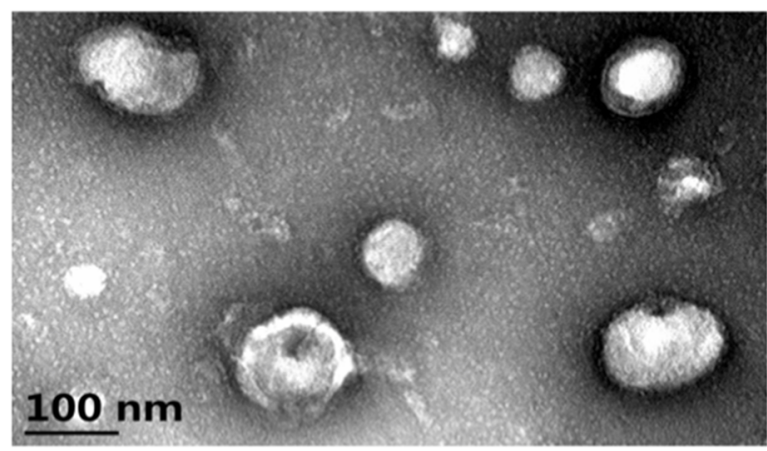

C

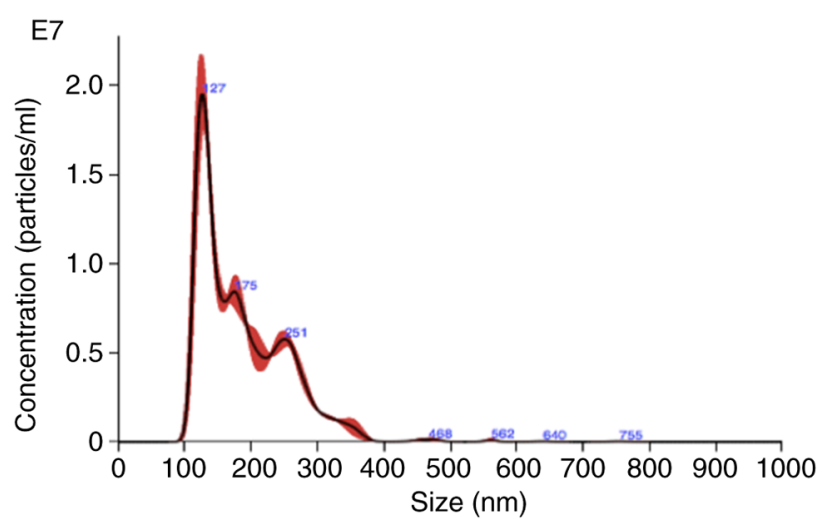

B

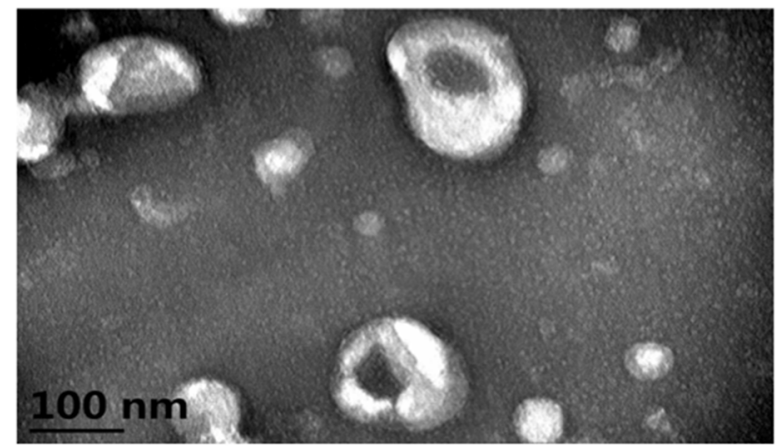

D

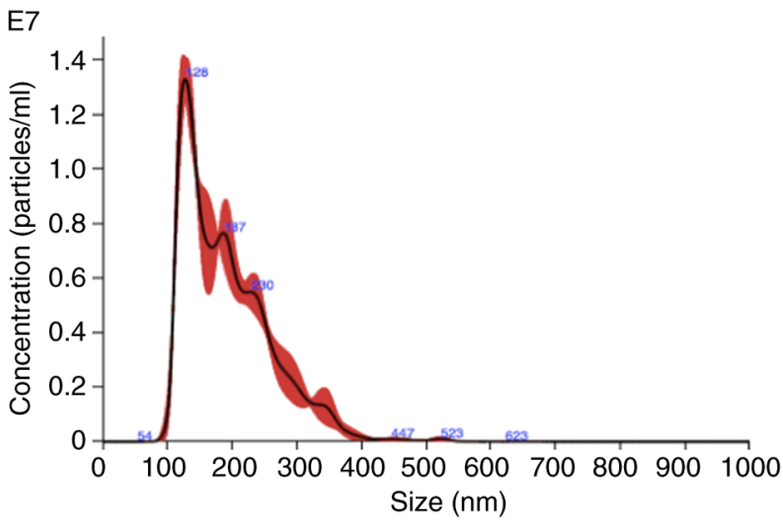

E

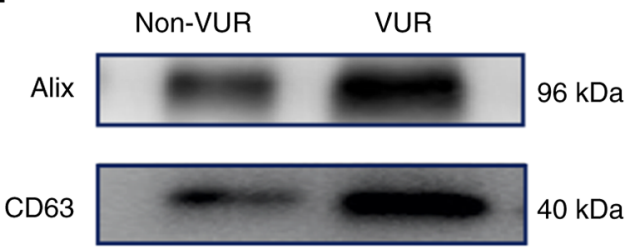

F

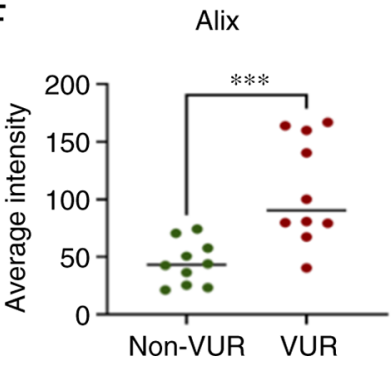

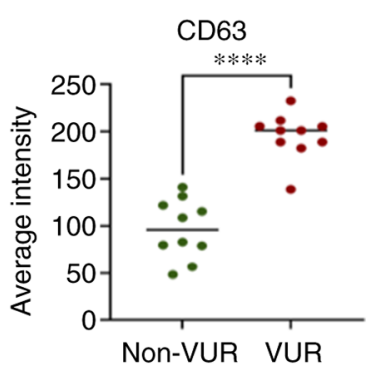

Figure 2. Characterization and identification of urinary exosomes. (A) Images of urinary exosomes under electron microscope from non-VUR patients. (B) Images of urinary exosomes under electron microscope from VUR patients. Scale bars, 100 nm. NTA results of urinary exosomes from (C) non-VUR and (D) VUR patients. (E) Expression of exosomal markers (Alix and CD63) by western blotting from isolated urinary exosomes of VUR and non-VUR groups. (F) Quantitative analysis of Alix and CD63, respectively. $\mathrm{n}=10$ for each group. ${ }^{* * *} \mathrm{P}<0.001,{ }^{* * * * *} \mathrm{P}<0.0001$. VUR, vesicoureteral reflux.

no significance between the two groups. In addition, a bladder safe capacity significantly $<300 \mathrm{ml}$ in the VUR group suggests that the effective bladder volume was shrinking, which is a sign of decreased bladder compliance and contracture.

The clinical data suggested that the bladder functional status was significantly different between the VUR and non-VUR groups, and worse lower urinary tract damage was observed in patients with VUR.

Urinary exosome characterization and identification. Exosomes were characterized using TEM, NTA and western blotting. Morphologically, exosomes appeared as spherical-like structureS surrounded by a layer of membrane-like material under TEM (Fig. 2A and B). NTA illustrated that the mean diameters of exosomes were $106.7 \pm 60.0 \mathrm{~nm}$ and $186.4 \pm 67.4 \mathrm{in}$ the non-VUR and VUR groups, respectively (Fig. 2C and D). Exosome markers Alix and CD63 were detected using western blotting, which confirmed that the effectiveness of exosome sample isolation. Notably, Alix and CD63 excretion were significantly enhanced in VUR patients compared with non-VUR patients (Fig. 2E and F).

Briefly, patients with VUR excreted exosomes with bigger diameters containing more exosome markers, such as Alix and CD63.

Proteomic profile and bioinformatics analysis. According to the TMT results, a total of 15,208.0 peptide segments were identified, of which the specific peptide segment was 14,648.0. The present study identified 2,622.0 proteins, of which 2,024.0 were quantifiable (Table SI). EV-specific proteins such as CD63, CD9 and Alix were expressed in all samples (Table SI). Compared with the non-VUR group, 134 protein expressions were upregulated and 99 protein expressions were downregulated in the VUR group with 1.5 -fold as the threshold of differential expression change and $\mathrm{P}<0.05$ as the significant threshold (Fig. 3A; Table SII). 

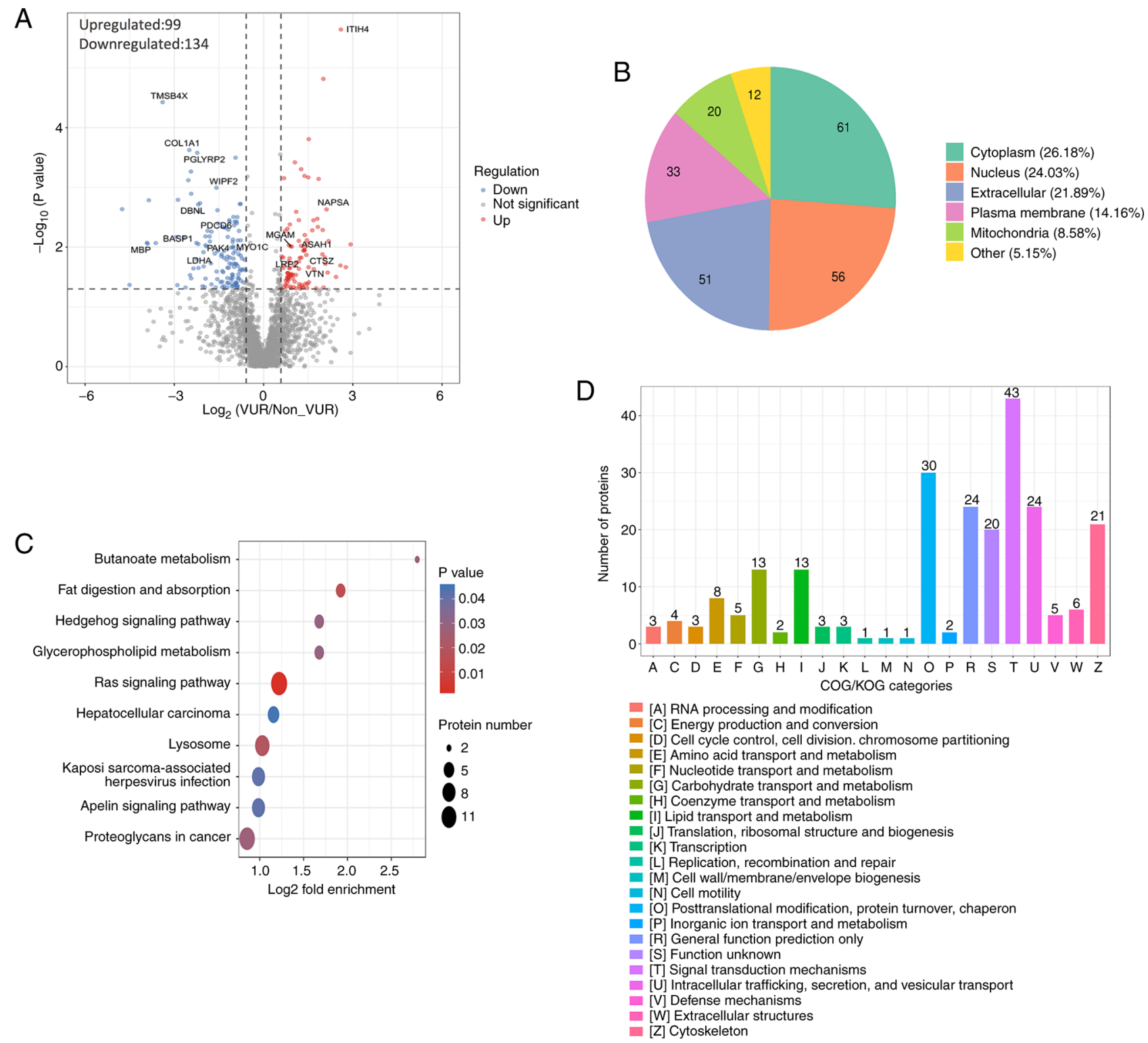

Figure 3. Results of bioinformatics analysis of identified proteins between the VUR and non-VUR groups. (A) Volcano plot showing the differentially expressed proteins between VUR and non-VUR groups. $n=5$ for each group in the mass spectrometry analysis. (B) Pie diagrams of Gene Ontology analysis of the differentially expressed proteins between VUR and non-VUR groups. (C) Kyoto Encyclopedia of Genes and Genomes pathway analysis. (D) Clusters of COG/KOG categories show functional annotation of differential proteins. VUR, vesicoureteral reflux; COG/KOG, Orthologous Groups/euKaryotic Ortholog Groups of proteins.

Subcellular structural localization indicated that $21.89 \%$ of differentially expressed proteins were localized extracellularly and $26.18 \%$ were located in the cytoplasm (Fig. 3B). Kyoto Encyclopedia of Genes and Genomes analysis revealed these differentially expressed proteins were mainly associated with the 'RAS signaling pathway' and 'lysosome' system, which are the most active pathways (Fig. 3C). For a deep study, COG/KOG category analysis reported that 43 proteins were associated with the signal transduction mechanisms (Fig. 3D). In addition, 24 proteins demonstrated a close relationship with intracellular trafficking, secretion and vesicular transport (Fig. 3D).

Based on the peak of peptide segments and bioinformatics analyses aforementioned, 18 proteins were selected for PRM verification. The molecular location and functions of the 18 candidate proteins were listed in Table II according to the
UniProt database. For example, thymosin $\beta$-4 (TMSB4X), WAS/WASL interacting protein family member 2 , unconventional myosin-Ic, drebrin-like protein and p21-activated kinase 4 (PAK4) were associated with the cytoskeleton. Other proteins were associated with apoptosis, regeneration or migration, such as brain acid soluble protein 1 (BASP1), VTN, cathepsin Z, L-lactate dehydrogenase A chain, programmed cell death protein 6 , PAK4, N-acylsphingosine amidohydrolase 1 and inter- $\alpha$-trypsin inhibitor heavy chain H4 (ITIH4). Moreover, the present study also revealed that BASP1 and low density lipoprotein-related protein 2 were directly associated with renal function, indicating possible abnormalities in renal function. The expression differences of these 18 candidate proteins are presented in the heatmap (Fig. 4A). GO enrichment analysis of 18 candidate markers suggested that the majority of functions were associated with 
Table II. Cell localization and biological function of the 18 candidate biomarkers.

\begin{tabular}{|c|c|c|}
\hline Name & Location & Molecular function \\
\hline BASP1 & Nucleus & Mesenchymal to epithelial transition, glomerular visceral epithelial cell differentiation \\
\hline COL1A1 & Extracellular & A member of the group I collagen \\
\hline VTN & Extracellular & Cell adhesion and spreading factor \\
\hline LRP2 & Plasma/membranee & Important for the functional integrity of the kidney \\
\hline MBP & Nucleus & The most abundant protein components of the myelin membrane in the CNS \\
\hline TMSB4X & Nucleus & $\begin{array}{l}\text { Plays an important role in the organization of the cytoskeleton and inhibits actin } \\
\text { polymerization }\end{array}$ \\
\hline WIPF2 & Nucleus & $\begin{array}{l}\text { Plays an active role in the formation of cell surface protrusions and reorganization of } \\
\text { the actin filament system }\end{array}$ \\
\hline CTSZ & Extracellular & Positive regulation of neuron apoptotic process, regulation of neuron death \\
\hline PGLYRP2 & Extracellular & Regulation of inflammatory response, innate immune response \\
\hline MGAM & Golgi apparatus & An alternate pathway for starch digestion \\
\hline MYO1C & Cytoplasm & $\begin{array}{l}\text { Regulating movement of intracellular vesicles to the plasma membrane. Links the actin } \\
\text { cytoskeleton to cellular membranes }\end{array}$ \\
\hline LDHA & Cytoplasm & Positive regulation of the apoptotic process \\
\hline PDCD6 & Cytoplasm nucleusus & $\begin{array}{l}\text { Plays a key role in endoplasmic reticulum-Golgi vesicular transport, endosomal } \\
\text { biogenesis or membrane repair. }\end{array}$ \\
\hline DBNL & Nucleus & $\begin{array}{l}\text { Plays a role in the reorganization of the actin cytoskeleton, formation of cell projections } \\
\text { and in neuron morphogenesis }\end{array}$ \\
\hline PAK4 & Nucleus & $\begin{array}{l}\text { Cytoskeleton regulation, cell migration, growth, proliferation or cell survival and } \\
\text { stabilization of actin filaments. }\end{array}$ \\
\hline ASAH1 & Extracellular & $\begin{array}{l}\text { Mediate cellular signaling pathways including cell proliferation, apoptosis and } \\
\text { differentiation }\end{array}$ \\
\hline GSK3A & Nucleus & Regulation of transcription factors and microtubules anti-apoptotic function \\
\hline ITIH4 & Extracellular & Involved in inflammatory responses to trauma and plays a role in regeneration \\
\hline
\end{tabular}

BASP1, brain acid soluble protein 1; COL1A1, $\alpha$-1 type I collagen; VTN, vitronectin; LRP2, low density lipoprotein-related protein 2; MBP, myelin basic protein; TMSB4X, thymosin $\beta$-4; WIPF2, WAS/WASL interacting protein family member 2; CTSZ, cathepsin Z; PGLYRP2, peptidoglycan recognition protein 2; MGAM, maltase-glucoamylase; MYO1C, unconventional myosin-Ic; LDHA, L-lactate dehydrogenase A chain; PDCD6, Programmed cell death protein 6; DBNL, Drebrin-like protein; PAK4, p21-activated kinase 4; ASAH1, N-acylsphingosine amidohydrolase 1; GSK3A, glycogen synthase kinase-3 $\alpha$; ITIH4, Inter- $\alpha$-trypsin inhibitor heavy chain H4.

the biological mechanism of exosomes, such as 'extracellular exosome' and 'vesicle-mediated transport' (Fig. 4B). The results also revealed that these proteins were significantly associated with, for example, 'vesicle', 'RAS' and 'lysosome' systems, 'extracellular matrix' and 'signaling pathway' (Fig. 4C). The present results were highly consistent with the bioinformatics results of 233 differential proteins in Fig. 3C. Furthermore, functions including 'fibrosis', 'transforming growth factor beta', 'focal adhesion' and 'smooth muscle actin' also showed strong associations with the present 18 candidate proteins (Fig. 4C). Among these proteins, it was revealed that the aforementioned functional enrichment results were mainly contributed to by VTN, myelin basic protein (MBP), COL1A1 and ITIH4 (Fig. 4C). The present results seemed to indicate that the four candidate proteins were more likely to predict disease progression. Hence, the PRM validation experiments were expected to corroborate with these results.

In the PRM validation, only 14 of the 18 proteins were quantitatively analyzed, while the remainder consisting of MBP, TMSB4X, peptidoglycan recognition protein 2 and glycogen synthase kinase-3 $\alpha$ were excluded because of failure to be quantified. PRM analysis revealed that only VTN $(\mathrm{P}=0.005)$, and COL1A1 $(\mathrm{P}=0.043)$ had significant differences between the two groups (Fig. 4D; Table SIII). Therefore, VTN and COL1A1 were selected for further validation.

Collectively, significantly different protein profiles of urinary exosomes were uncovered between the VUR and non-VUR groups. Signal transduction-related and vesicle-related proteins represented the most significant proteins, especially VTN and COL1A1.

Validation by western blotting. As presented in Fig. 5, exosomal proteins VTN and COL1A1 were both detected using western blotting despite VTN expression being significantly stronger compared with COL1A1. Total protein was set as the loading control to ensure comparability between the two groups (Fig. 5A). The intensity of the bands for each protein was then compared with the total protein, and the ratios are presented in Fig. 5C. Both exosomal VTN $(\mathrm{P}=0.0003)$ and COL1A1 $(\mathrm{P}=0.042)$ were significantly higher in VUR patients compared with those in non-VUR patients (Fig. 5B-D). The variation 
A

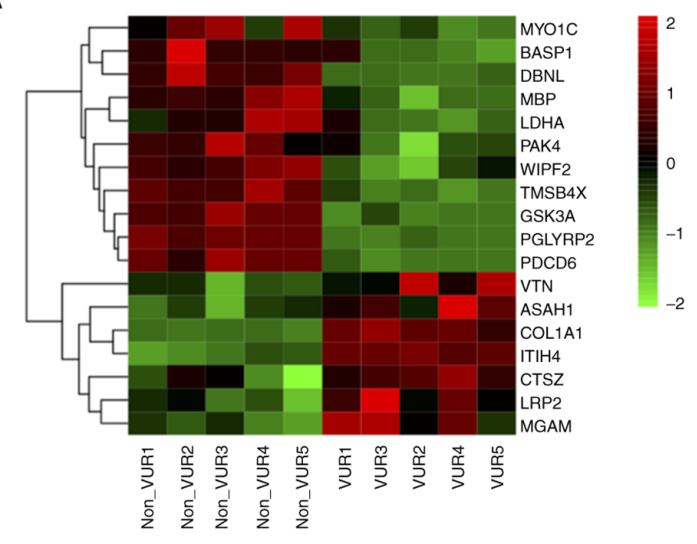

C

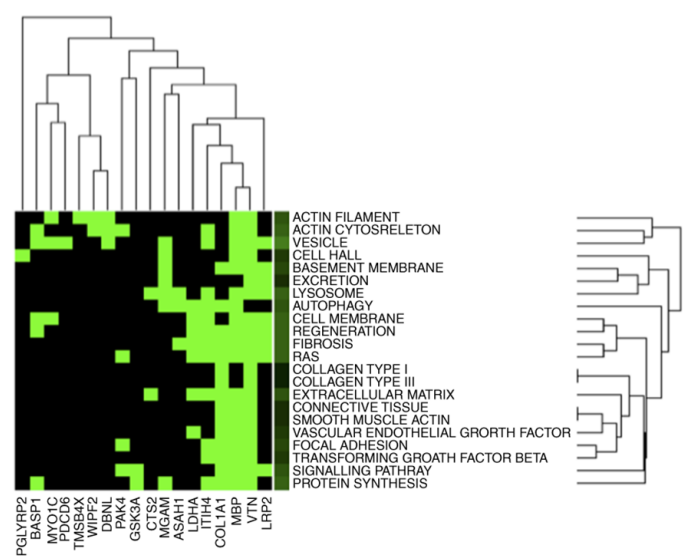

B

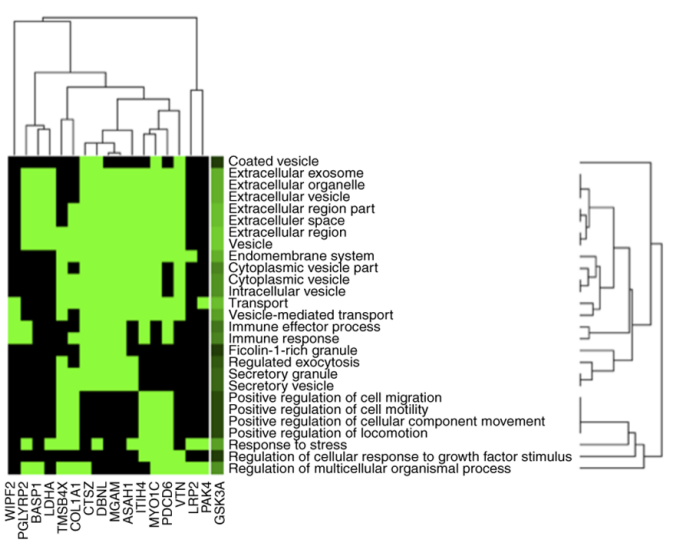

D

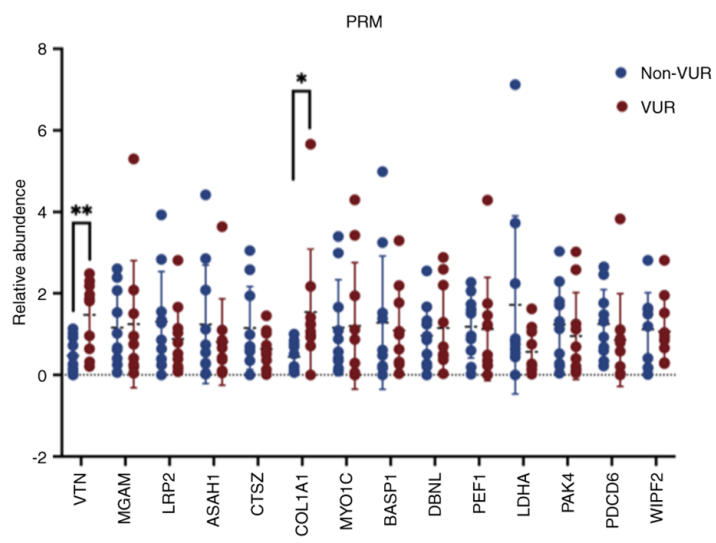

Figure 4. Bioinformatics analysis and PRM results for the 18 candidate proteins. (A) Expression differences of 18 candidate proteins. (B) Gene Ontology enrichment analysis of 18 candidate markers by GenCLiP 3.022. (C) Gene function enrichment (FunRich) analysis of 18 candidate markers by GenCLiP 3.022 . (D) Relative peak area of 18 candidate proteins was measured by PRM between VUR and non-VUR groups. $\mathrm{n}=5$ for each group in the PRM analysis. ${ }^{*} \mathrm{P}<0.05$, ${ }^{* *} \mathrm{P}<0.01$. PRM, parallel reaction monitoring; VUR, vesicoureteral reflux.

tendency of exosomal VTN and COL1A1 between VUR and non-VUR patients was very similar to that observed by TMT.

The present results suggested that patients with VUR excreted more VTN and COL1A1 compared with non-VUR patients, and that VTN and COL1A1 in urinary exosomes may be potential diagnostic markers for predicting VUR.

ELISA validation and ROC. ELISA results demonstrated that the VTN concentrations in patients with VUR were significantly higher compared with those in the non-VUR patients $(\mathrm{P}<0.0001$; Fig. 6A). However, no significance was revealed in exosomal COL1A1 between the two groups by ELISA ( $\mathrm{P}=0.1499$; Fig. 6B). It was suggested that COL1A1 may not be stably expressed in exosomes. Accordingly, only the ELISA results of the VTN were used to generate the ROC curve analysis. The ROC curve had an AUC of 0.795 (95\% CI, 0.667-0.923) for VTN, with $80 \%$ sensitivity at $82.9 \%$ specificity (Fig. 6C).

Overall, the above data demonstrated that urinary exosomal VTN could distinguish and predict VUR in patients with NGB.

\section{Discussion}

The present study identified the differences in urine exosomal protein profiles between VUR and non-VUR patients and revealed that exosomal VTN could be used as a potential marker in predicting disease progression in patients with NGB. At present, there are no recognized non-invasive, effective biochemical indicators to predict urinary reflux (25). The present results demonstrated that urine exosomal VTN may be an early biomarker for monitoring VUR.

Decreases in bladder compliance and bladder remodeling are the main causes of urine reflux (26). Studies have indicated that bladder overfilling and abnormally high pressure can lead to bladder structure injury and an increase in bladder wall thickness $(27,28)$. NGB causes a loss of normal urination function, resulting in long-term overfilling and abnormally high pressure of bladder tissue, damaged bladder tissue structure, compensatory thickening of smooth muscle, increased deposition of collagen fibers in bladder tissue and eventually leads to bladder tissue fibrosis (29-32). Based on these findings, the present study hypothesized that the increase of urine exosomal VTN may indicate a poor prognosis of bladder fibrosis and remodeling.

VTN, also known as S-protein, is one of the main components of the extracellular matrix that participates in cell spreading (33), cell adhesion (34) and cell migration (35). VTN is highly associated with various types of tissue fibrosis $(36,37)$, including the fibrosis of kidney disease (38). More recently, a study focused on urine exosomal VTN revealed a positive relationship with renal fibrosis (39). Urine exosomal VTN may 
A

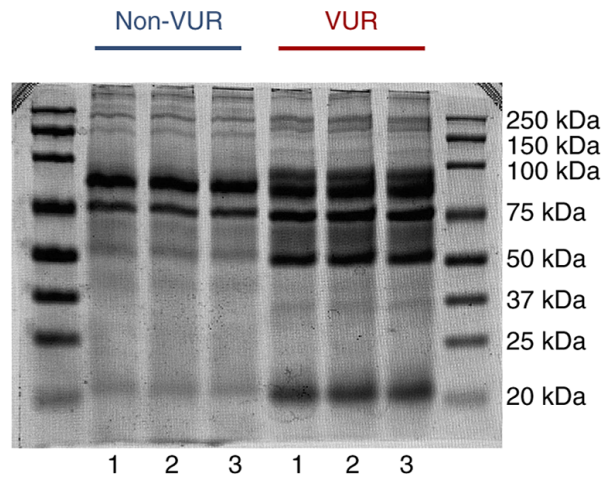

C

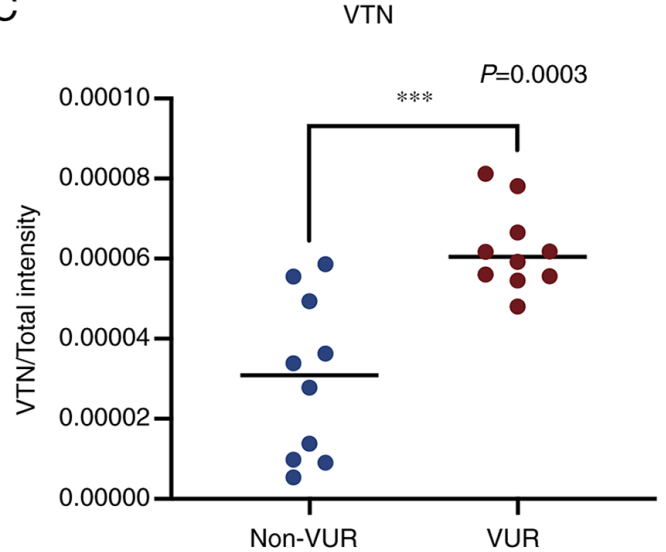

B

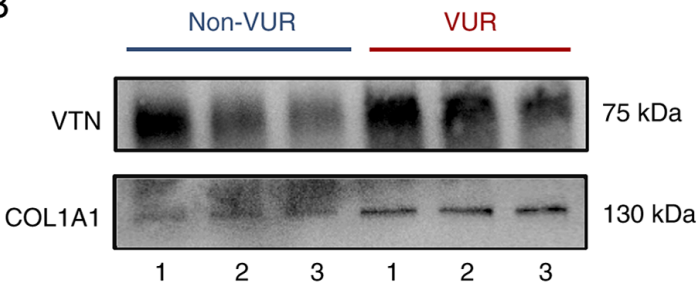

D

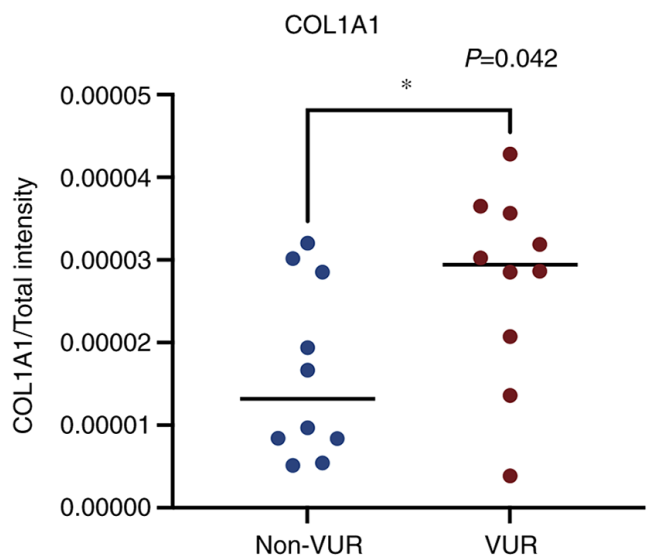

Figure 5. Western blotting detection of VTN and COL1A1 between VUR and non-VUR groups. (A) Western blotting results from similar loaded amounts of proteins stained by Coomassie G250 methods. (B) Western blotting results of VTN and COL1A1 when similar amounts of proteins were loaded between VUR and non-VUR groups. (C) VTN/total protein intensity ratio between VUR and non-VUR groups. (D) COL1A1/total protein intensity ratio between VUR and non-VUR groups. $\mathrm{n}=10$ for each group. ${ }^{* * *} \mathrm{P}<0.001,{ }^{*} \mathrm{P}<0.05$. VUR, vesicoureteral reflux; COL1A1, $\alpha-1$ type I collagen; VTN, vitronectin.

A

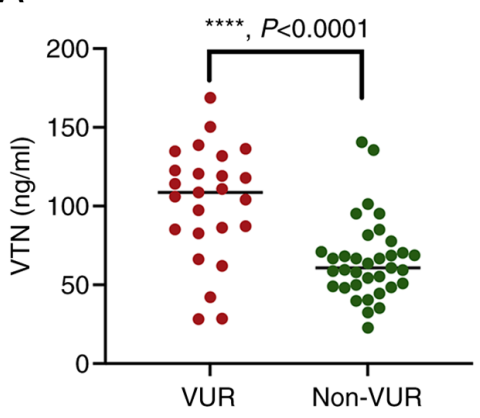

B

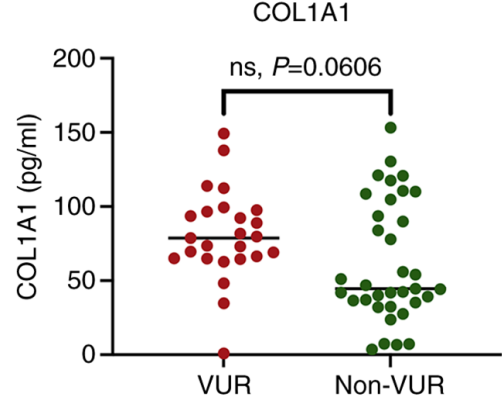

C

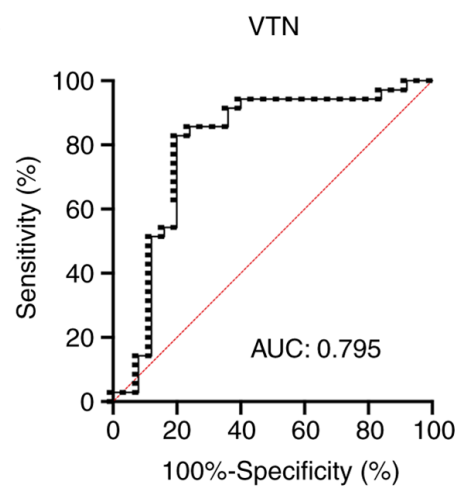

Figure 6. ELISA results of VTN and COL1A1 between VUR and non-VUR groups. (A) ELISA result of VTN between VUR and non-VUR groups. (B) ELISA result of COL1A1 between VUR and non-VUR groups. (C) Receiver operating curve analysis of VTN between VUR and non-VUR groups. $\mathrm{n}=25$ for the VUR group and $\mathrm{n}=35$ for the non-VUR group. ${ }^{* * * * *} \mathrm{P}<0.0001$. n.s., not significant; VUR, vesicoureteral reflux; COL1A1, $\alpha-1$ type I collagen; VTN, vitronectin; AUC, area under curve.

be secreted from the kidney or the bladder. However, to the best of our knowledge, no studies have demonstrated that VTN is associated with bladder fibrosis at present. The present study firstly proposed that exosomal VTN is highly associated with NGB fibrosis, instead of renal fibrosis. The main reasons are as follows: i) Bladder remodeling and fibrosis caused by bladder dysfunction are the main pathological mechanisms of bladder compliance decline; ii) the decrease in bladder compliance causes urine reflux and kidney damage and, as a result, bladder fibrosis and remodeling should be an earlier warning event of urine reflux. Consequently, it was hypothesized that elevated VTN may be more associated with bladder fibrosis compared with renal fibrosis. Furthermore, VTN mediates tissue fibrosis progression by upregulating TGF- $\beta 1$ (40). Activation of TGF- $\beta /$ Smad signaling pathways appear to be an important mechanism of tissue fibrosis (41), including in the kidney (42) and bladder (43). Consistent with this theory, the present study also revealed that fibrosis and TGF- $\beta$ signaling pathways were 
highly activated. However, whether urine exosomal VTN also promotes fibrosis through the TGF- $\beta /$ Smad signaling pathway in NGB requires further investigation.

According to the TMT results, the expression of COL1A1 was significantly downregulated. However, its expression was significantly elevated in the VUR group as demonstrated by PRM and western blotting validation. Moreover, the significant difference disappeared when ELISA was performed. It was hypothesized that the possible explanations for these contradictory results mainly include the three following reasons. First, the different sample sizes may contribute to those inconsistencies, as only five cases in each group were used in the mass spectrometry analysis experiment while 10 samples were used in the PRM validation experiments. When ELISA was performed among the 60 included samples, the difference of COL1A1 between groups disappeared. Therefore, COL1A1 was not selected to be a candidate biomarker. Accordingly, it was hypothesized that this was directly associated with sample size and individual differences between samples. Second, these may be associated with different progression statuses of diseases. COL1A1 is a part of type I collagen, which is an important component of the extracellular matrix (ECM). Abnormal ECM deposition is a major pathological change in fibrosis (44). That is to say that COL1A1 would be significantly elevated when significant fibrosis exists in the bladder wall. In the present study, patients were grouped by urine reflux. The patients with significant fibrosis were included in the VUR group, which resulted in great individual variability of COL1A1 in the VUR group. Consistent with this interference, the ELISA results of COL1A1 in the VUR group revealed high variability. Thirdly, the results of the ELISA validation may be limited by the sensitivity of the ELISA kits. Accordingly, future studies will need to increase the sample sizes and develop more cost-efficient and sensitive kits.

Although COL1A1 was not selected to be an effective biomarker, it may still be associated with disease progression. Functional enrichment analysis in the present study revealed that extracellular matrix-related functions were also significantly activated. Collagen I and III are the main factors of bladder compliance (45). Once collagen is heavily deposited in the bladder wall, elastin becomes relatively reduced, leading to thicker bladder walls, decreased smooth muscle function, reduced bladder compliance and even bladder fibrosis (46). Moreover, the present study revealed that the RAS pathway was significantly activated. The RAS-MAPK pathway is an important mechanism for causing epithelial-mesenchymal transition and has a significant role in the development of fibrosis (47). Although the final ELISA results were not satisfactory, COL1A1 still may play a notable role in the decline of bladder compliance and bladder fibrosis, and may be used as a biological marker to predict the occurrence of VUR. Following studies should focus on the relevance of COL1A1 to disease progression.

Several limitations should be noted in the present study. First, the sample size was limited, and bigger cohort studies are required to confirm these observations. Secondly, exosomal COL1A1 revealed no significance in the ELISA validation. These may be limited by the sensitivity of the ELISA kits. Therefore, developing a more cost-efficient and sensitive method for the detection of proteins is important and worthwhile. Third, exosomal VTN was identified to be an effective biomarker for the diagnosis of VUR. Whether VTN may be associated with the progression of bladder remodeling and fibrosis is still an important factor and this is another limitation of the present study. According to the aforementioned evidence of VTN and COL1A1 being involved in bladder remodeling, subsequent studies should further explore the impact of different degrees of damage on the profile of exosome secretion. Additionally, future studies will investigate whether urine exosomal VTN is involved in bladder remodeling and fibrosis via the TGF- $\beta / \mathrm{Smad}$ signaling pathway in NGB.

Overall, to the best of our knowledge, the present study proposed for the first time that the increase of urinary exosomal VTN could be a potential marker in predicting VUR in patients with NGB. Alterations in these exosomal proteins may suggest early urinary tract remodeling. The mechanism of VTN (perhaps including COL1A1) involved in urinary tract remodeling needs to be verified, and a large-scale prospective study is needed.

\section{Acknowledgements}

The authors would like to thank Professor Xin Li, chief director of Central Laboratory of Shenzhen Hospital, Southern Medical University (Shenzhen, China), for providing the rabbit anti-CD63 antibody.

\section{Funding}

This work was supported by Sanming Project of Medicine in Shenzhen, China (grant no. SZSM201612018).

\section{Availability of data and materials}

The datasets used and/or analyzed during the current study are available from the corresponding author on reasonable request.

\section{Authors' contributions}

WC conceived the initial idea, designed the study and revised the manuscript. JL wrote the manuscript and performed the majority of the experiments. SC and CZ collected the urinary samples and performed some of the experiments. LC, CZ and $\mathrm{YH}$ were responsible for the data analysis and conception of this article. JL and SC confirmed the authenticity of all the raw data. All authors have read and approved the final manuscript.

\section{Ethics approval and consent to participate}

The study protocol was approved by the Scientific Ethics Committee of Shenzhen Hospital, Southern Medical University (approval no. NYSZYYEC20180002; Shenzhen, China). All participants provided written informed consent according to the principles of the Helsinki Declaration.

\section{Patient consent for publication}

Not applicable. 


\section{Competing interests}

The authors declare that they have no competing interests.

\section{References}

1. Ahuja CS, Wilson JR, Nori S, Kotter MRN, Druschel C, Curt A and Fehlings MG: Traumatic spinal cord injury. Nat Rev Dis Primers 3: 17018, 2017.

2. Cristante AF, Barros Filho TE, Marcon RM, Letaif OB and Rocha ID: Therapeutic approaches for spinal cord injury. Clinics (Sao Paulo) 67: 1219-1224, 2012

3. Furlan JC, Noonan V, Singh A and Fehlings MG: Assessment of impairment in patients with acute traumatic spinal cord injury: A systematic review of the literature. J Neurotrauma 28: 1445-1477, 2011.

4. Noreau L, Noonan VK, Cobb J, Leblond J and Dumont FS: Spinal cord injury community survey: A national, comprehensive study to portray the lives of canadians with spinal cord injury. Top Spinal Cord Inj Rehabil 20: 249-264, 2014

5. Dave CN, Khalaf A, Patel HD, Kohn TP and Burnett AL: Neurogenic bladder is an independent risk factor for complications associated with inflatable penile prosthesis implantation. Int J Impot Res 32: 520-524, 2020

6. Li LF, Ka-Kit Leung G and Lui WM: Sacral nerve stimulation for neurogenic bladder. World Neurosurg 90: 236-243, 2016.

7. Nseyo U and Santiago-Lastra Y: Long-term complications of the neurogenic bladder. The Urol Clin North Am 44: 355-366, 2017.

8. Zhang T, Liu H, Liu Z and Wang L: Acupuncture for neurogenic bladder due to spinal cord injury: A systematic review protocol. BMJ Open 4: e006249, 2014

9. Théry C, Zitvogel L and Amigorena S: Exosomes: Composition, biogenesis and function. Nat Rev Immunol 2: 569-579, 2002.

10. Dai J, Su Y, Zhong S, Cong L, Liu B, Yang J, Tao Y, He Z, Chen C and Jiang Y: Exosomes: Key players in cancer and potential therapeutic strategy. Signal Transduct Target Ther 5: 145, 2020.

11. He C, Zheng S, Luo Y and Wang B: Exosome theranostics: Biology and translational medicine. Theranostics 8: 237-255, 2018

12. Chen C, Luo Y, He W, Zhao Y, Kong Y, Liu H, Zhong G, Li Y Li J, Huang J, et al: Exosomal long noncoding RNA LNMAT2 promotes lymphatic metastasis in bladder cancer. J Clin Invest 130: 404-421, 2020.

13. Cimadamore A, Gasparrini S, Santoni M, Cheng L, Lopez-Beltran A, Battelli N, Massari F, Giunchi F, Fiorentino M, Scarpelli $\mathrm{M}$ and Montironi R: Biomarkers of aggressiveness in genitourinary tumors with emphasis on kidney, bladder, and prostate cancer. Expert Rev Mol Diagn 18: 645-655, 2018.

14. Quaglia M, Merlotti G, Guglielmetti G, Castellano G and Cantaluppi V: Recent advances on biomarkers of early and late kidney graft dysfunction. Int J Mol Sci 21: 5404, 2020.

15. Dear JW, Street JM and Bailey MA: Urinary exosomes: A reservoir for biomarker discovery and potential mediators of intrarenal signalling. Proteomics 13: 1572-1580, 2013.

16. Zhou H, Pisitkun T, Aponte A, Yuen PS, Hoffert JD, Yasuda H, Hu X, Chawla L, Shen RF, Knepper MA and Star RA: Exosoma Fetuin-A identified by proteomics: A novel urinary biomarker for detecting acute kidney injury. Kidney Int 70: 1847-1857, 2006.

17. Feng Y, Lv LL, Wu WJ, Li ZL, Chen J, Ni HF, Zhou LT, Tang TT, Wang FM, Wang B, et al: Urinary exosomes and exosomal CCL2 mRNA as biomarkers of active histologic injury in IgA nephropathy. Am J Pathol 188: 2542-2552, 2018.

18. Mizutani K, Kawakami K, Horie K, Fujita Y, Kameyama K, Kato T, Nakane K, Tsuchiya T, Yasuda M, Masunaga K, et al: Urinary exosome as a potential biomarker for urinary tract infection. Cell Microbiol 21: e13020, 2019.

19. Roberts TT, Leonard GR and Cepela DJ: Classifications in brief: American spinal injury association (ASIA) impairment scale. Clin Orthop Relat Res 475: 1499-1504, 2017

20. Wang JH, Zhao LF, Wang HF, Wen YT, Jiang KK, Mao XM Zhou ZY, Yao KT, Geng QS, Guo D and Huang ZX: GenCLiP 3: Mining human genes' functions and regulatory networks from PubMed based on co-occurrences and natural language processing. Bioinformatics: Nov 4, 2019 (Epub ahead of print).

21. Jeppesen DK, Fenix AM, Franklin JL, Higginbotham JN, Zhang Q, Zimmerman LJ, Liebler DC, Ping J, Liu Q, Evans R, et al: Reassessment of exosome composition. Cell 177: 428-445.e18, 2019
22. Eaton SL, Roche SL, Llavero Hurtado M, Oldknow KJ, Farquharson C, Gillingwater TH and Wishart TM: Total protein analysis as a reliable loading control for quantitative fluorescent western blotting. PLoS One 8: e72457, 2013

23. Wang L, Skotland T, Berge V, Sandvig K and Llorente A Exosomal proteins as prostate cancer biomarkers in urine: From mass spectrometry discovery to immunoassay-based validation. Eur J Pharm Sci 98: 80-85, 2017.

24. RStudio Team: RStudio: Integrated development for R. RStudio, Inc., Boston, MA, 2015.

25. Valério FC, Lemos RD, de C Reis AL, Pimenta LP, Vieira ÉL and Silva ACE: Biomarkers in vesicoureteral reflux: An overview. Biomark Med 14: 683-696, 2020.

26. Wu CQ and Franco I: Management of vesicoureteral reflux in neurogenic bladder. Investig Clin Urol 58 (Suppl 1): S54-S58, 2017.

27. Ekiz A, Özdemir-Kumral ZN, Erşahin M, Tuğtepe H, Öğünç AV, Akakın D, Kiran D, Özsaveı D, Biber N, Hakan T, et al: Functional and structural changes of the urinary bladder following spinal cord injury; treatment with alpha lipoic acid. Neurourol Urodyn 36: 1061-1068, 2017.

28. Ozsoy O, Ozsoy U, Stein G, Semler O, Skouras E, Schempf G, Wellmann K, Wirth F, Angelova S, Ankerne J, et al: Functional deficits and morphological changes in the neurogenic bladder match the severity of spinal cord compression. Restor Neurol Neurosci 30: 363-381, 2012

29. Hamid R, Averbeck MA, Chiang H, Garcia A, Al Mousa RT, Oh SJ, Patel A, Plata M and Del Popolo G: Epidemiology and pathophysiology of neurogenic bladder after spinal cord injury. World J Urol 36: 1517-1527, 2018.

30. Ginsberg D: The epidemiology and pathophysiology of neurogenic bladder. Am J Manag Care 19 (Suppl 10): s191-s196, 2013.

31. Hu HZ, Granger N and Jeffery ND: Pathophysiology, clinical importance, and management of neurogenic lower urinary tract dysfunction caused by suprasacral spinal cord injury. J Vet Intern Med 30: 1575-1588, 2016

32. Compérat E, Reitz A, Delcourt A, Capron F, Denys P and Chartier-Kastler E: Histologic features in the urinary bladder wall affected from neurogenic overactivity-a comparison of inflammation, oedema and fibrosis with and without injection of botulinum toxin type A. Eur Urol 50: 1058-1064, 2006.

33. Preissner KT and Reuning U: Vitronectin in vascular context: Facets of a multitalented matricellular protein. Semin Thromb Hemost 37: 408-424, 2011.

34. Madsen CD and Sidenius N: The interaction between urokinase receptor and vitronectin in cell adhesion and signalling. Eur J Cell Biol 87: 617-629, 2008

35. Naik MU and Naik UP: Junctional adhesion molecule-A-induced endothelial cell migration on vitronectin is integrin alpha $\mathrm{v}$ beta 3 specific. J Cell Sci 119: 490-499, 2006.

36. Shen TL, Liu MN, Zhang Q, Feng W, Yu W, Fu XL and Cai XW: The positive role of vitronectin in radiation induced lung toxicity: The in vitro and in vivo mechanism study. J Transl Med 16: 100, 2018.

37. Hayashida M, Hashimoto $\mathrm{K}$, Ishikawa $\mathrm{T}$ and Miyamoto $\mathrm{Y}$ : Vitronectin deficiency attenuates hepatic fibrosis in a nonalcoholic steatohepatitis-induced mouse model. Int J Exp Pathol 100: 72-82, 2019.

38. López-Guisa JM, Rassa AC, Cai X, Collins SJ and Eddy AA: Vitronectin accumulates in the interstitium but minimally impacts fibrogenesis in experimental chronic kidney disease. Am J Physiol Renal Physiol 300: F1244-F1254, 2011

39. Carreras-Planella L, Cucchiari D, Cañas L, Juega J, Franquesa M Bonet J, Revuelta I, Diekmann F, Taco O, Lauzurica R and Borràs FE: Urinary vitronectin identifies patients with high levels of fibrosis in kidney grafts. J Nephrol 34: 861-874, 2021.

40. Ciregia F, Deroyer C, Cobraiville G, Plener Z, Malaise O, Gillet P, Fillet M, Malaise MG and de Seny D: Modulation of $\alpha_{V} \beta_{6}$ integrin in osteoarthritis-related synovitis and the interac-

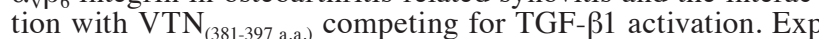
Mol Med 53: 210-222, 2021

41. Su J, Morgani SM, David CJ, Wang Q, Er EE, Huang YH, Basnet H, Zou Y, Shu W, Soni RK, et al: TGF- $\beta$ orchestrates fibrogenic and developmental EMTs via the RAS effector RREB1. Nature 577: 566-571, 2020.

42. Ma TT and Meng XM: TGF- $\beta /$ Smad and renal fibrosis. Adv Exp Med Biol 1165: 347-364, 2019.

43. Chen Y, Ma Y, He Y, Xing D, Liu E, Yang X, Zhu W, Wang Q and Wen JG: The TGF- $\beta 1$ pathway is early involved in neurogenic bladder fibrosis of juvenile rats. Pediatr Res 90: 759-767, 2021. 
44. Alyaseer AAA, de Lima MHS and Braga TT: The role of NLRP3 inflammasome activation in the epithelial to mesenchymal transition process during the fibrosis. Front Immunol 11: 883, 2020.

45. Liu C, Wan X, Gu M, Chen Y, Cai Z, Zhou J, Chen Q and Wang Z: Effect of sulforaphane on bladder compliance in a rat model of partial bladder outlet obstruction. Oxid Med Cell Longev 2019: 6026719, 2019.

46. Kraft M, Oussoren Y, Stewart FA, Dörr W and Schultz-Hector S: Radiation-induced changes in transforming growth factor beta and collagen expression in the murine bladder wall and its correlation with bladder function. Radiat Res 146: 619-627, 1996.
47. Feng YL, Chen DQ, Vaziri ND, Guo Y and Zhao YY: Small molecule inhibitors of epithelial-mesenchymal transition for the treatment of cancer and fibrosis. Med Res Rev 40: 54-78, 2020.

cc)(i) (9) This work is licensed under a Creative Commons Attribution-NonCommercial-NoDerivatives 4.0 International (CC BY-NC-ND 4.0) License. 